\title{
Immune Parameters in High-risk Atopic Individuals during Early Childhood
}

\author{
HUUB F. J. SAVELKOUL \\ Department of Immunology, Erasmus University Rotterdam, Rotterdam, The Netherlands
}

\section{ALLERGIC ASTHMA IS A Th2 DISEASE}

The profile of cytokine production by peripheral blood mononuclear cells (PBMCs) from patients with bronchial asthma on stimulation with house dust mite was demonstrated to be compatible with that of helper T type 2 (Th2) cells (1). The number of Th2 cells was also shown to be increased in bronchial tissue and bronchoalveolar lavage fluid of patients with bronchial asthma. Also, the recruitment of allergen-specific Th2 cells from the peripheral blood into the bronchial tissue after an inhalant allergen challenge was demonstrated. Allergic asthma is therefore considered a Th2-driven disease in which the presence of Th 2 cells correlates with IgE production, mast cell hyperplasia, and eosinophil infiltration, determining the severity of the disease (2). The Th2-derived cytokine interleukin 5 (IL-5) is a crucial cytokine, which accounts for the recruitment and activation of eosinophils, resulting in airway eosinophilia in patients with allergy (3). In animal models, depletion of $\mathrm{CD}^{+} \mathrm{T}$ cells results in abrogation of airway eosinophilia and in subsequent reduction of airway hyperresponsiveness. Similarly, elevated IL-4-induced serum levels of IgE and allergen-specific IgE levels as observed in patients with atopic allergy can be explained in terms of excessive Th2 activation. Many, but not all, the pleiotropic effects of IL-4, which are of importance in allergic diseases, can be inhibited by interferon $\gamma$ (IFN- $\gamma$ ). Thus, the balance between Th1/Th2 activation is generally established by determining the ratio between IL-4 and IFN- $\gamma$ produced by activated PBMCs or purified T cells.

\section{COGNATE INTERACTION AND CYTOKINE-INDUCED ISOTYPE SWITCHING}

IgE isotype switching in B lymphocytes requires physical interaction of Th cells and B cells via surface molecules (primarily CD40-CD40 ligand [CD40L]) and the presence of IL-4 and/or IL-13 secreted by Th2 cells (4). Immunoglobulin class switching is generally considered a two-step process. In the first step, the downstream target isotype-specific heavy chain constant $\left(\mathrm{C}_{\mathrm{H}}\right)$ gene locus becomes transcriptionally active, resulting in the synthesis of germline transcripts. This transcriptional activity has been proposed to reflect the opening up of the $\mathrm{C}_{\mathrm{H}}$ locus, rendering it accessible to IL-4 and IL-13. The IL-4 promotor contains multiple binding sites for transcription factors, including the STAT- 6 transcription induced by both IL-4 and IL-13. The second step in IgE switching requires the CD40 molecule. Antigen-activated T-B cell cognate interactions result in Th cell activation and expression of the CD40L, facilitating interaction with $\mathrm{CD} 40^{+} \mathrm{B}$ cells that induces prolif-

Correspondence and requests for reprints should be addressed to H. F. J. Savelkoul, M.D., Erasmus University Rotterdam, Department of Immunology, P.O. Box 1738, 3000DR Rotterdam, The Netherlands. E-mail: savelkoul@immu.fgg.eur.nl

Am J Respir Crit Care Med Vol 162. pp S100-S104, 2000

Internet address: www.atsjournals.org eration and differentiation in an antigen-independent fashion. The CD40 signal provides a strong proliferation signal, which furthermore prevents the activated cells from undergoing apoptosis. Likewise, human B cells stimulated with IL-4 in the presence of CD40 cross-linking secrete detectable amounts of $\mathrm{IgG}_{4}$ approximately $2 \mathrm{~d}$ earlier than $\mathrm{IgE}$. On the basis of such data it is hypothesized that the switch from $\operatorname{IgM}$ to $\operatorname{IgE}$ typically involves an initial switching to $\mathrm{IgG}_{4}(5)$. The relevance of this sequential switching in vivo remains to be established.

The activating effect of IL-4 is promoted by IL-5 and IL-6, but inhibited by IFN- $\gamma$, transforming growth factor $\beta$ (TGF- $\beta$ ), and prostaglandin $\mathrm{E}_{2}\left(\mathrm{PGE}_{2}\right)$. Likewise, these mediators inhibit IL-4-induced expression of CD23 on B cells, indicating that there is an association between CD23 expression and IL-4-induced IgE production. Furthermore, IL-10 has a downmodulating effect on the production of IFN- $\gamma$. In T and B cell cocultures of patients with an established allergy, the IgE production was more dependent on endogenously produced IL-13 than was the IgE production by control subjects, whereas IL-4 neutralization resulted in equal inhibition of IgE production in patients and control subjects. The contribution of IL-4 and IL- 13 to allergen-specific IgE production of human B cells in vivo remains to be determined.

In allergic diseases it can be assumed that the abnormally high levels of IgE antibodies in response to allergens are derived from $\mathrm{B}$ cells that are abnormally stimulated or respond abnormally to an allergenic stimulus. It has been observed that when B cells from children with atopic diseases are cultured in vitro, they spontaneously synthesized 5,000 pg of IgE per $10^{6}$ cells, whereas the same number of B cells from healthy controls synthesized only $250 \mathrm{pg}$. It is as yet unresolved whether this difference is due to an increased precursor frequency of IgE-secreting B cells, an increased clone size, or an increased production rate per cell.

\section{IL-4 AND IL-4 RECEPTORS IN ATOPY}

IL-4 has a critical role in the regulation of differentiation of naive T cells into Th2 cells and the upregulation of IgE synthesis. IL-4 is encoded on chromosome $5 \mathrm{q} 31.1$ in close proximity to other cytokine genes (IL-3, IL-5, IL-9, IL-13, and granulocyte-macrophage colony-stimulating factor [GM-CSF]) contributing to the development of allergic inflammation and IgE production. Genetic polymorphisms have been identified within the regulatory elements of the IL-3, IL-4, and IL-9 genes belonging to the IL-4 gene cluster. The transcription of the IL-4 gene is regulated by multiple promoter elements that either induce or suppress transcription. Thus, it has been demonstrated that the human IL-4 promotor region exists in multiple allelic forms, which differ in the level of IL-4 transcription and expression in T cells. Therefore, it is generally agreed that people with asthma produce abnormally high amounts of IL-4 (6).

Currently, two different IL-4 receptors have been described, one of which contains the 140-kD IL-4-binding protein (IL-4R $\alpha / \mathrm{CDw} 124)$, which dimerizes with the common $\gamma$ 
chain shared with the IL-2, IL-7, IL-9, IL-13, and IL-15 receptor. Furthermore, IL-4R $\alpha$ is able to associate with one of the chains of the IL-13 receptor that comprises the type II IL-4 receptor. The role of the type II receptors on different cell types is still the subject of research. The presence of the common $\gamma$ chain in the type I receptor complex increases the binding affinity of IL-4 by two- to threefold and contributes to signal transduction, including activation of the STAT-6 transcription factor. A genetic polymorphism in the intracellular signaling part of the IL-4 receptor has been identified whereby normal levels of IL-4 may exert stronger biological effects resulting in higher levels of, for example, IgE (7). This altered signaling function of the receptor is strongly associated with atopy and the underlying mutation may predispose susceptible persons to allergic diseases.

\section{ALLERGIC SENSITIZATION IN EARLY LIFE}

It is generally appreciated that the prevalence of asthma is increasing, and currently asthma affects one child in seven in Western Europe (8). The reasons for this apparent increase are suggested to include a better and earlier diagnosis, more efficient treatment regimens, a shift from severe to more mild disease, and an increased exposure to environmental allergens. The T cell memory that develops against allergens is currently believed to be the result of complex interactions between genetic susceptibility and environmental factors. Such factors as parental smoking, (viral) infections, (breast) feeding methods, birthweight, elevated total IgE levels in cord blood, early exposure to household pets, and inhalant allergens have all been implicated to increase the risk of the development of atopic disorders (2). The prevalence of atopic diseases in socalled developed countries has risen over the past several decades, which is too short a period for genetic change to take place. Therefore, the environmental influence is much larger than the hereditary component.

It has been proposed that the fetal immune system is weakly skewed toward the preferential development of Th2type immune responses, as a result of the production of Th2promoting factors, such as IL-10 and $\mathrm{PGE}_{2}$, by the placenta ( 9 , 10). Such an environment is, however, similar both for atopyprone and health nonatopic children. For the expansion and increased activity of these Th2 cells, intrauterine allergen exposure would be required. It is not understood how and to what extent allergens can pass the placental barrier. It has been recognized before that the placental barrier is not merely a molecular sieve with a defined pore size, but is actively able to transfer sometimes large molecules into the fetus. In the transfer of specific IgG antibodies, the placenta could selectively transfer antibodies on the basis of their idiotypic specificity.

After birth, house dust-mite specific stimulation revealed a persisting Th2 response in atopic children, while nonatopic children developed a gradual transition toward a preferential Th1 response driven by the traditionally frequent exposure to mycobacteria. In the Western world, lack of exposure to mycobacteria could be responsible for the increasing failure of inducing such a Th1 development and, consequently, can explain the rise in atopic diseases observed over the last $25 \mathrm{yr}$. Also, other observations suggesting a decline in viral and bacterial infections since the introduction of vaccination and the development of antibiotics has resulted in a limitation of Th1 immune mechanisms and induced Th2 polarization, resulting in an increasing prevalence of atopic diseases. On the basis of in vitro analysis, selective outgrowth of a Th2 population could be based on a preferential expansion of Th2 cells or the relative absence or the selective inhibition of the activity of Th1 cells. Evidence has led to both mechanisms being described as playing a role in the development of allergic asthma after birth. It remains to be established whether the increased prevalence of allergic asthma needs to be explained in the cytokine-dependent Th1-Th2 polarization or whether more shifts from Th2 ${ }^{\text {low }}$ to Th $2^{\text {high }}$ activity may be sufficient. Also, the suggested decreased interferon $\gamma($ IFN- $\gamma$ ) production in atopic relative to nonatopic children needs to be confirmed. The consequence of the absence of IFN- $\gamma$ would be a decreased allergen-specific delayed type hypersensitivity (DTH) response in allergic versus nonallergic individuals, which could not be found. The role of IFN- $\gamma$ in atopic diseases is therefore important beyond the mere Th1-Th2 paradigm.

Whereas the risk of contracting atopic disease during childhood is between 15 and $20 \%$ in the general population, it is increased to 35 to $40 \%$ if the child's mother has an atopic disease. The risk with two atopic parents increases to about $60 \%$. Children born to atopic mothers therefore constitute a highrisk population and are well suited for the study of the importance of age-related changes in immune parameters that are implicated in the expression of atopic diseases at the neonatal stage. Although asthmatic diseases can start at any age, in more than $50 \%$ of cases asthma symptoms start before the age of $3 \mathrm{yr}$. The early onset of asthma determines the persistence of the disease into adulthood, and is therefore considered an important risk factor. Other important risk factors include exposure to cigarette smoke, male sex, and viral infections, including respiratory syncytial virus and parainfluenza virus. It has been suggested that the immaturity of the immune system together with completion of lung development at the neonatal age might contribute to the mechanism of early atopic sensitization.

\section{IMMATURITY OF THE NEONATAL IMMUNE SYSTEM}

Accumulating evidence suggests that the immune system of newborns is relatively immature as compared with adults, which may fit into the concept of a window period with increased susceptibility to develop atopy $(11,12)$. The capacity of cord blood $\mathrm{T}$ cells to produce cytokines in response to allergen-specific stimulation such as IFN- $\gamma$ is strongly diminished, which might be due to the presence of $\mathrm{PGE}_{2}$, an intrinsic deficiency in the synthesis capacity, or an inefficient accessory cell function. The lack of sufficient IFN- $\gamma$ production may contribute to impaired neonatal antiviral responses and facilitate allergic sensitization as the result of abnormal $\mathrm{T}$ cell function. The Th2 cytokines were either expressed too weakly (IL-4) or did not differ significantly (IL-5, IL-10). The secretion of immunoglobulins by B cells is strongly decreased while the number of $\mathrm{CD} 23^{+} \mathrm{B}$ cells is increased in newborns relative to adults. This might explain the enhanced negative feedback regulation of $\operatorname{IgE}$ formation at the neonatal stage.

Moreover, antigen-presenting cells (APCs) are functionally immature, related to their impaired phagocytosis and chemotaxis. Although it had originally been shown that high-dose antigen exposure results in Th2 responses, atopic individuals have a genetically based propensity to react with IgE antibody formation to low doses of allergens that do not result in antibody formation in nonatopic subjects. On activation APCs produce a plethora of cytokines that influence $\mathrm{T}$ cell polarization. Monocytes of atopic individuals have been shown to produce increased levels of $\mathrm{PGE}_{2}$ and reduced levels of IL-12. The cognate interaction between the APC and the Th cell, however, is complex and involves also the timing and strength of costimulatory molecules, the strength of the interaction 
with the $\mathrm{T}$ cell receptor, and temporal changes in the production of cytokines. What steps are crucially different between atopic and nonatopic individuals has not been elucidated yet.

The percentages of peripheral blood $\mathrm{CD}^{+} \mathrm{T}$ cells expressing the activation markers CD25 and HLA-DR (human leukocyte antigens) and $\mathrm{CD}^{+} \mathrm{T}$ cells expressing CD25 were elevated in children with severe asthmatic disease. It is unclear whether children with mild atopic disease display similar shifts in expression of surface markers. Cord blood $\mathrm{T}$ cells display no or decreased expression of CD40L on stimulation, which together with the failure to express costimulatory cytokines (IL-5, IL-6) might explain the poor immunoglobulin production in the neonate. Most neonatal CD4 ${ }^{+} \mathrm{T}$ cells are immunologically naive and express CD45RA (91\%). These cells are gradually replaced by $\mathrm{CD} 45 \mathrm{RO}$, a marker of activated or memory $\mathrm{T}$ cells, as a result of repeated allergen exposure. The latter cells are considered to be the high-rate cytokine-secreting cells. In atopic adults, the percentage of CD45RO ${ }^{+} \mathrm{T}$ cells was significantly elevated compared with healthy control subjects. Whether the same holds true for atopic children is not known in detail.

\section{THE INDUCTION OF TOLERANCE}

As outlined above, helper $\mathrm{T}$ cell subset polarization is achieved by two basic principles. First, each subset produces its own autocrine growth factors and, second, cytokines are produced that cross-regulate the outgrowth of the counteracting subset. However, the observed skewing in subjects with allergic asthma to the Th2 compartment may also result from other regulatory processes. The preferential activation of Th2 cells could be the result of active suppression of the Th1 subset due to the cytokine-related interaction between $\mathrm{T}$ cell subsets and the APCs. Unresponsiveness can be achieved by different mechanisms including clonal deletion by apoptosis, by T cell anergy, or by active suppression mediated by regulatory cells. These mechanisms are not mutually exclusive, but may act synergistically.

The process of $\mathrm{T}$ cell anergy is best defined as a state of cellular unresponsiveness in which the cell is alive but unable to perform functional responses on restimulation. Anergized cells are generated by antigen-specific $\mathrm{T}$ cell activation in the absence of costimulatory molecules. This partial activation signal renders these cells unable to proliferate and to secrete IL-2. The main contributors of the costimulatory signals are CD28 on the T cell and B7-1 (CD80) and B7-2 (CD86) on the APC. This interaction enhances the transcription rate and stabilization of IL-2 mRNA and increases the sensitivity of T cell receptor (TCR) triggering. The $\mathrm{B} 7$ molecules possess still another ligand, CTLA-4. This ligand has been shown to negatively regulate $\mathrm{T}$ cell responses and to evoke a complete block in the cell cycle progression and IL-2 production. CD28 expression on the $\mathrm{T}$ cell is constitutive, whereas CTLA-4 is induced early after $\mathrm{T}$ cell activation. The differences in affinities and binding kinetics with B7-1 and B7-2 determine whether a cell becomes activated or remains unresponsive. The precise role of APCs in tolerance induction is yet to be determined.

Clonal deletion of antigen-specific $\mathrm{T}$ cells and anergy are both passive mechanisms regulating immune responses. The ability to regulate helper $\mathrm{T}$ cell responses is important with regard to Th1 cytokine production, because it has been shown that excessive and sustained Th1 cytokine production may provoke deleterious proinflammatory responses. Activation-induced apoptosis is mediated by interaction of Fas ligand (FasL) with its cognate receptor Fas. Th1 cells express high levels of FasL, while Th2 cells express only low levels. Therefore, Th1 cells are more sensitive to apoptosis and this property limits continuous expansion of a proinflammatory Th1 response.

Regulatory $\mathrm{T}$ cells, on the other hand, mediate active suppression $(13,14)$. These $\mathrm{T}$ cells comprise classic Th2 cells, capable of inhibiting Th1 responses, but also alternative $\mathrm{T}$ cell populations. The mechanism of peripheral tolerance has been focused mainly on the suppression of classic cell-mediated type 1 responses and on animal models of diseases based on excessive activity of Th1 cells. It is now clear that such tolerance induction is also active in humoral type 2 responses, such as IgE production. Suppression becomes apparent after administration of allergens via the oral, respiratory, and nasal routes. One of the primary mechanisms of tolerance induction is via secretion of immunosuppressive cytokines such as IL-10, IL-4, and transforming growth factor $\beta$ (TGF- $\beta$ ). As mentioned above, regulatory $\mathrm{CD} 4^{+} \mathrm{T}$ cell clones, isolated from in vitro cultures, have appeared to produce low levels of IL-2 and no IL-4, but high levels of IL-10 and TGF- $\beta$. When cocultured with naive $\mathrm{CD}^{+} \mathrm{T}$ cells, these antigen-specific clones suppressed the proliferative response to the same antigen. The regulatory $\mathrm{T}$ cell clones themselves exhibited a low proliferative capacity. Neutralization of IL-10 and TGF- $\beta$ overcomes this proliferative block. The activity of TGF- $\beta$-secreting regulatory $\mathrm{T}$ cells has been implicated as a component of oral tolerance, in recovery from experimental allergic encephalomyelitis, and in suppression of certain forms of inflammatory colitis. TGF- $\beta$-deficient mice experience inflammation that affects multiple organ systems, mainly the heart, liver, and lungs, whereas IL-10 knockout mice produce a chronic enterocolitis. This demonstrates the importance of these cytokines in regulating and dampening the immune response.

Human counterparts of regulatory $\mathrm{T}$ cells have been proposed in patients with severe combined immunodeficiency (SCID), who after bone marrow reconstitution revealed high numbers of IL-10 (but not IL-4)-producing $\mathrm{CD}^{+}$cells in which IL-10 levels were associated with tolerance after allogenic stem cell transplantation. At present, it is unknown whether these regulatory $\mathrm{T}$ cells are involved in the immunopathology of allergic asthma by provoking excessive suppression of the Th1 compartment.

\section{CHEMOKINES AND ADHESION MOLECULES IN ATOPIC DISEASES}

Soluble factors, including chemokines, play an essential role in leukocyte trafficking toward and infiltration of the airways of individuals with asthma. CC chemokines have been implicated in the immunopathology of allergic asthma by their chemotactic and stimulatory activity and their ability to induce degranulation and histamine release from basophils. The $\mathrm{CC}$ chemokines RANTES (regulated on activation, normal $T$ cell expressed and presumably secreted) and monocyte chemoattractant protein (MCP)-3 act on eosinophils, whereas MCP-1, MCP-3, and RANTES affect basophil functions. Some reports have described chemotactic proteins responsible for directing $\mathrm{Th} 2$ lymphocytes toward allergic airways. Interestingly, the major contributor to this process is not a traditional chemokine, but the interleukin IL-16 (15). This interleukin is released in response to allergens, mitogen, histamine, or serotonin by $\mathrm{CD}^{+}$ $\mathrm{T}$ cells, eosinophils, and airway epithelial cells and possibly $\mathrm{CD}^{+} \mathrm{T}$ cells. IL-16 chemotactically attracts $\mathrm{CD} 4^{+} \mathrm{T}$ cells, eosinophils, and monocytes by interacting with the CD4 molecule. In patients with asthma, IL-16 was detected in the bronchoalveolar lavage (BAL) fluid $6 \mathrm{~h}$ after allergen challenge. In fact, $80 \%$ of the chemotactic activity is ascribed to IL-16, whereas the remaining activity is due to the CC chemokine 
macrophage inflammatory protein $1 \alpha$ (MIP- $1 \alpha)$. The involvement of eotaxin in the process of Th2 trafficking has been proposed as well. It was shown that after in vitro polarization of $\mathrm{CD} 4^{+} \mathrm{T}$ cells, the Th2-polarized cells showed a higher expression of the eotaxin ligand CCR3. Hypothetically, this implies that once eotaxin is released within inflamed airways, Th2 cells (but not Th1 cells) are selectively attracted toward the site of inflammation, resulting in progressive polarization of the local Th2 cell response.

Adhesion molecules, such as intracellular adhesion molecule 1 (ICAM-1) and E-selectin, the membrane-bound form of soluble E-selectin, are cell surface adhesion molecules that are expressed on endothelial cells during inflammatory processes and determine leukocyte recruitment and trafficking into inflamed tissues (16). Therefore, the soluble forms of these adhesion molecules, generated by alternative splicing or proteolytic cleavage, generally correlate with the degree of inflammatory infiltrates, thereby providing useful parameters for monitoring the disease severity and successful therapeutic interventions. In asthma, increased bronchial epithelium expression of ICAM-1 was found with a concomitant presence of increased serum levels of soluble ICAM-1. Despite these finding the role of ICAM in the pathogenesis of asthma remains to be established.

\section{CONCLUSION}

It has been proposed that expression of Th2-polarized immunity to inhaled (environmental) allergens at the level of the airway mucosa is typical of the pathogenesis of atopic respiratory disease. At birth, there is evidence of a resting, but previously (in utero) activated, memory $\mathrm{T}$ cell population that could be responsible for the rapid release of cytokines after neonatal allergen exposure. Generally, this neonatal atopic predisposition is expressed within the first 1 to $2 \mathrm{yr}$ of life. During this period, the immune system is also gradually maturing and is highly flexible. This property potentially results in the induction of clinical symptoms of atopic disease, the allergy window period. Gradually the immune system establishes control over the neonatal immune responses, which can take the first $5 \mathrm{yr}$ of life. However, it remains to be established to what degree expression of food allergic symptoms during the first year of life is related to the probability of developing inhalation allergy at ages above $3 \mathrm{yr}$, as there are conflicting data published. These findings open possibilities to link immunopathological changes evident in the first few years of life to the development of asthma-like symptoms extending into adulthood. Such research is, however, dependent on the unequivocal diagnosis of asthma at the age of 5-6 yr. Developments have made essential lung function measurements possible at this age and this facilitates proper asthma diagnosis.

\section{References}

1. Romagnani, S. 1994. Lymphokine production by human T cells in disease state. Annu. Rev. Immunol. 12:227-257.

2. Koning, H., M. R. M. Baert, A. P. Oranje, H. F. J. Savelkoul, and H. J. Neijens. 1996. Development of immune functions, related to allergic mechanisms, in young children. Pediatr. Res. 40:363-375.

3. Corrigan, C. J., and A. B. Kay. 1992. T cells and eosinophils in the pathogenesis of asthma. Immunol. Today 13:501-507.

4. Cocks, B., R. de Waal Melefyt, J. P. Galizzi, J. E. de Vries, and G. Aversa. 1993. IL-13 induces proliferation and differentiation of human B cells activated by the CD40Ligand. Int. Immunol. 5:657-663.

5. Mills, F. C., G. Thyphronitis, F. D. Finkelman, and E. E. Max. 1992. Immunoglobulin M-E isotype switch in IL-4 treated human B lymphoblastoid cells: evidence for a sequential switch. J. Immunol. 149:10751085.

6. Marsh, D. G., J. D. Neely, D. R. Breazeale, B. Ghosh, L. R. Freidhoff, E.
Ehrlich-Kautzky, C. Schou, G. Krishnaswamy, and T. H. Beatty. 1994. Linkage analysis of IL-4 and other chromosome 5q31.1 markers and total IgE concentrations. Science 264:1152-1156.

7. Hershey, G K. K., M. F. Friedrich, L. A. Esswein, M. L. Thomas, and T. A. Chatila. 1997. The association of atopy with a gain of function mutation in the $\alpha$ subunit of the IL-4 receptor. N. Engl. J. Med. 337: $1720-1725$.

8. Tariq, S. M., S. M. Matthews, E. A. Hakim, M. Stevens, S. H. Arshad, and D. W. Hide. 1998. The prevalence of and risk for atopy in early childhood: a whole population birth cohort study. J. Allergy Clin. Immunol. 101:587-593.

9. Wegmann, T. G., H. Lin, L. Guilbert, and T. R. Mosmann. 1993. Bidirectional cytokine interactions in the maternal-fetal relationship: is successful pregnancy a Th phenomenon? Immunol. Today 14:353-356.

10. Prescott, S. L., C. Macaubas, B. J. Holt, T. B. Smallacombe, R. Itoh, P. D. Sly, and P. G. Holt. 1998. Transplacental priming of the human immune system to environmental allergens: universal skewing of initial $\mathrm{T}$ cell responses toward the Th2 cytokine profile. J. Immunol. 60 : $4730-4737$.

11. Lewis, D. B., C. Yu, C. Meyer, B. K. English, S. Kahn, and C. B. Wilson. 1991. Cellular and molecular mechanisms for reduced interleukin-4 and interferon- $\gamma$ production by neonatal T cells. J. Clin. Invest. 87: 194-202.

12. Splawski, B., and P. E. Lipsky. 1991. Cytokine regulation of immunoglobulin secretion by neonatal lymphocytes. J. Clin. Invest. 88:967-977.

13. O'Garra, A., L. Steinman, and K. Gijbels. 1997. CD4 ${ }^{+}$T cell subsets in autoimmunity. Curr. Opin. Immunol. 9:872-883.

14. Thomas, M. J., and D. M. Kemeny. 1998. Novel CD4 and CD8 T cell subsets. Allergy 53:1122-1132.

15. Center, D. M., H. Kornfeld, and W. W. Cruikshank. 1996. Interleukin 16 and its function as a CD4 ligand. Immunol. Today 17:476-481.

16. Takahashi, N., M. C. Liu, D. Proud, X. Y. Yu, S. Hasegawa, and E. W. Spannhake. 1994. Soluble intracellular adhesion molecule 1 in bronchoalveolar lavage fluid of allergic subjects following segmental antigen challenge. Am. J. Respir. Crit. Care Med. 150:704-709.

\section{DISCUSSION}

Holt: We too observed that it is key to be extremely careful in handling of the cells up to the final processing. We have worked out a protocol that involved putting the cells into an appropriate transport medium. We shipped cells regularly between Perth and Melbourne and Dr. Björkstén is using the same system to ship cells between Dublin and Linköping. It is important to do the appropriate controls, but that part of the story is solvable. With respect to the defect in interferon $\gamma$ production, this is not an absolute defect. You don't see an effect until you look at the relative levels of production as a function of age. Would you agree with that?

Savelkoul: Yes. We observed the IFN- $\gamma$ defect in some children, but it is certainly not absolute. The decreased IL-10 levels we see in combination with the increased IL-4 expression are very interesting, since these cells clearly are not Th2 cells. The decreased IL-10 production resembles that in IFN- $\gamma$ production, although we don't know whether it is based on the same intrinsic defect.

We observe this defect under some conditions, but not in all children. The point I want to make is that there is variability. How that is regulated we don't know. There might be differences in chaperone proteins that help in the transport over the cell membrane.

Holt: We are also becoming very interested in IL-10. We have been looking at the relationship between the magnitude in the skin test response in children at various ages in relation to the size of the IL-10 response and indeed we see a very strong negative relationship, which would argue just as you have done that there is important negative feedback in vivo by IL-10.

Savelkoul: I fully agree with that. Under many conditions you find after stimulation a little bit of IL-4 and a lot of IL-10. People tend to dichotomize the T cell system and say: These are Th 2 cells. However, it takes 3 or 4 days before these cells are 
generated in vitro. So, initially they need IL-4 and finally they may produce only IL-10. If one uses a single stimulus with a heterogenous population of cells, it is too easy to propose a $\mathrm{Th} 2$ response after reading out three or four cytokines that are associated with the Th2 phenotype.

Björkstén: We can also confirm the IL-10 story. We see high levels of IL-10 in atopic dermatitis patients with negative skin prick tests, while the IL-4 levels are similar in skin test-positive and negative subjects. you found that the cord blood cells were activated. Is this the birth in itself? Have you studied babies born by caesarean section?

Savelkoul: No. We do not know what is due to the phenotype and what is induced by the stress during birth. I agree that birth has a profound influence on cytokine levels, but this cannot easily explain the observed shift in the cellular composition of the cord blood.

Vercelli: The presence of $\varepsilon$ germline transcripts in B cells of nonallergic or not-yet allergic children as well as in B cells of allergic children implies a two-hit model for IgE production. What is the second hit that would take the cells from the expression of $\varepsilon$ germline transcripts to actually switching?

Savelkoul: We don't know. We have to examine more kids. In these studies only 10-15 children were analyzed, but in the cohort of Dr. Van der Laan we have cells available to run this in a much larger set of children. We would also like to study $\operatorname{IgG}_{4}$ germline transcripts.

Out: You showed that $\mathrm{T}$ cells in cord blood from the high-risk babies were more activated than those from controls. Yesterday, Dr. Holt showed us that $\mathrm{T}$ cell clones were generated in a lower frequency from the cord blood from babies that became atopic in later life and that these clones produced lower amounts of IL- 4 and IFN- $\gamma$. Do you consider this as a differ- ence between your results and his? And to Dr. Holt: Could it be an explanation that the already-activated cells do not grow out into $\mathrm{T}$ cell clones, thereby missing the activated cells in this analysis?

Savelkoul: You have to remember that the data I showed you are after polyclonal stimulation, while Dr. Holt's data are after antigen-specific stimulation. We have also accumulated antigen-specific stimulation data, but we are still processing these, so I can't answer now.

Platts-Mills: Since the cord blood cells are so active, how can you be sure that allergen-specific responses is not actually a mitogeneic response?

Savelkoul: That is very true. That will be very hard to segregate. Maybe Dr. Holt could comment.

Platts-Mills: It has always been difficult to understand why there should be a good allergen-specific response in the cord blood and at 6 months it disappears.

Holt: We don't see all this activation. So, all I can suggest is that it may be due to major differences between the populations that we are looking at, $75-85 \%$ of our kids are delivered by caesarean section; we have never looked at the obvious comparisons. But, I don't think that is it, because we just do not see high backgrounds in terms of proliferation. When we are talking about looking at allergen-specific responses we are talking about $100 \mathrm{dpm}$ as our background in terms of proliferation. Maybe it is Yssel's medium that is the problem in that context. It has never been an issue with us.

Savelkoul: I cannot exclude that. Hopefully, the allergen-specific data will tell us more. However, I don't think it is something purely technical, as we have found the increased population of $\mathrm{CD}^{+} \mathrm{CD}^{+} 5 \mathrm{RA}^{+}$in cord blood as well. 